Pacific Journal of Mathematic 


\title{
COMPLETE NON-SELFADJOINTNESS OF ALMOST SELFADJOINT OPERATORS
}

\author{
Thomas L. KRIETE
}

Suppose that $\alpha$ is a real-valued measurable function defined on the unit interval $[0,1]$ and that $c$ is a function in the Lebesgue space $L^{2}(0,1)$. Let $A$ be the (not necessarily bounded) operator on $L^{2}(0,1)$ associated with the pair $(\alpha, c)$ by

$$
(A f)(x)=\alpha(x) f(x)+i c(x) \int_{0}^{x} \overline{c(t)} f(t) d t .
$$

$A$ has the domain

$$
\mathscr{D}(A)=\left\{f \in L^{2}(0,1): \int_{0}^{1}|\alpha(x) f(x)|^{2} d x<\infty\right\}
$$

which is dense in $L^{2}(0,1)$. One easily verifies that the imaginary part $(2 i)^{-1}\left(A-A^{*}\right)$ extends to the bounded operator $f \rightarrow 1 / 2\langle f, c\rangle c$. Thus $A$ is almost selfadjoint in the sense that it differs from its real part by an operator of rank one.

The operators $A$ are more general than they appear. Livsic showed that every bounded operator $B$ with real spectrum, no selfadjoint part, and with nonnegative imaginary part of rank one is unitarily equivalent to the completely non-selfadjoint part of such an operator $A$ acting on $L^{2}(0, a)$ for some positive $a$. This raises the question of when (in terms of $\alpha$ and $c) A$ is completely non-selfadjoint. The main result of this paper answers this question when the pair $(\alpha, c)$ is subject to a mild restriction that is always satisfied when $A$ is bounded.

One consequence (Corollary 3.18) is a negative result concerning the behavior of singular spectral multiplicity under compact perturbations.

We need to establish some conventions and terminology. All Hilbert spaces throughout will be separable. Let $B$ be a densely defined operator on a Hilbert space $H$ with domain $\mathscr{D}(B)$. We will say that a subspace $N$ of $H$ reduces $B$ if $\mathscr{D}(B) \cap N$ and $\mathscr{D}(B) \cap N^{\perp}$ are dense in $N$ and $N^{\perp}$, respectively, and $B(\mathscr{D}(B) \cap N) \subset N$ and $B(\mathscr{D}(B) \cap$ $\left.N^{\lrcorner}\right) \subset N^{\perp}$. $B$ is said to be completely non-selfadjoint if the only reducing subspace $N$ for $B$ with the property that the restriction $B \mid N$ is selfadjoint is the zero subspace.

$B$ is dissipative if $\operatorname{Im}\langle B f, f\rangle \geqq 0$ for all $f$ in $\mathscr{D}(B)$. If in addition $(B+i / 2) \mathscr{D}(B)=H$, then $B$ is called maximal dissipative. In this case the Cayley transform $C=(B-i / 2)(B+i / 2)^{-1}$ is a contraction defined on all of $H$. (We have replaced $i$ by $i / 2$ in the Cayley 
transform to make some subsequent equations appear more natural.) There exists a unique reducing subspace $N$ for $C$ with the property that $C \mid N$ is unitary and $C \mid N^{\perp}$ is completely non-unitary. $N$ also reduces $B, B \mid N$ is selfadjoint, and $B \mid N^{\perp}$ is completely non-selfadjoint. Again $N$ is unique with respect to these properties (see [15]).

In $\S 3$ we will see that $A$ is maximal dissipative. To solve the problem at hand, it thus suffices to find the completely non-unitary part of $T=(A-i / 2)(A+i / 2)^{-1}$

We now set down the condition on the pair $(\alpha, c)$ that is needed to make our proof work. Suppose that $m$ denotes Lebesgue measure on $[0,1]$. Let $\nu$ be the measure on $(-\infty, \infty)$ given by

$$
\nu(F)=\int_{\alpha^{-1}(F)}|c|^{2} d m
$$

for every Borel subset $F$ of the reals. We denote Lebesgue measure on $(-\infty, \infty)$ by $n . d \nu / d n$ is the Radon-Nikodym derivative of $\nu$ with respect to $n$. We will demand that

$$
\int_{-\infty}^{\infty} \log \frac{d \nu}{d n}(x) \frac{d x}{x^{2}+1 / 4}=-\infty .
$$

Since $\{x: d \nu / d n(x) \neq 0\} \subset$ closed support of $\nu \subset$ essential range of $\alpha$, it is clear that (1.1) holds whenever the essential range of $\alpha$ (which is a closed set) is not all of $(-\infty, \infty)$. In particular, (1.1) holds if $A$ is bounded.

In the next section we write down some necessary information about Sz.-Nagy-Foias operator models and characterize a certain type of invariant subspace. An operator model operator $S$ acting on a space $K$ is then associated with the pair $(\alpha, c)$. In $\S 3$ we show that when (1.1) holds, it is possible to construct an isometry $W: K \rightarrow L^{2}(0,1)$ which gives a unitary equivalence between $S$ and the completely non-unitary part of $T=(A-i / 2)(A+i / 2)^{-1}$. We then give a criterion for deciding when $W$ is unitary, i.e., when $W K$ is all of $L^{2}(0,1)$. Since $A$ is completely non-selfadjoint provided $W K=L^{2}(0,1)$, this answers the question posed above. In $\S 4$ our methods are used to study almost unitary contractions with no isometric part.

A few remarks on the general spirit of this paper may be useful to the reader. Every completely non-unitary contraction $T_{0}$ acting on a separable Hilbert space $H$ is unitarily equivalent to an operator model $S$ in the sense of Sz.-Nagy and Foias [15, Chap. VI]. $S$ acts on a model Hilbert space $K . \quad T_{0}$ is determined up to unitary equivalence by the characteristic operator function $b$ of $S$. One knows the model theory for $T_{0}$ if one can specify $b$. Adopting terminology suggested by 
Douglas N. Clark, we will say that we know a concrete model theory for $T_{0}$ if we can specify $b$ together with an explicit unitary operator $U: H \rightarrow K$ with $U T_{0}=S U$. This is necessarily a little vague since the usual method for constructing $S$ from $T_{0}$ always yields an abstract form for $U$. What we mean here is that $U$ must be defined in terms of some additional structure that $H$ may possess as, say, a space of functions.

This paper offers an example of a concrete model theory with an application to a non-model-theoretic problem. We will take $T_{0}$ and $U$ to be, respectively, the restrictions $T \mid W K$ and $W^{*} \mid W K$ where $T$ and $W$ are as above. The model theory of $T \mid W K$ was known (modulo Cayley transforms) to Brodskii and Livsic [3], although they did not associate an operator model $S$ with the characteristic operator function. Perhaps the first example of a concrete model theory along these lines is due to Sarason [12] and, independently, to Rosenblum (unpublished). They considered the case in which $T$ is a function of the Volterra operator; the operator $U$ in this case is essentially a part of the Fourier transform. The present paper may be viewed as a natural extension of this work. Other examples of concrete model theories are given by the author [11], Ahern and Clark [1] and Clark [4].

From the point of view of model theory our most interesting result is probably Theorem 2 which relates the range of $W$ to the regularity (in the sense of Sz.-Nagy and Foias) of certain factorizations of $b$. These results were announced in [10].

I wish to thank Professor Marvin Rosenblum for suggesting a research problem that led to these results.

2. The operator $S$. Let $\sigma$ Lebesgue measure on the unit circle $\boldsymbol{T}$ in the complex plane normalized so that $\sigma(\boldsymbol{T})=1$. We sometimes consider $\sigma$ as a measure on $[0,2 \pi) . \quad \chi$ is the identity function on $\boldsymbol{T}: \chi\left(e^{i x}\right)=e^{i x}$. $\quad D$ will denote the open unit disk $\{z:|z|<1\}$.

If $1 \leqq p \leqq \infty, L^{p}=L^{p}(d \sigma)$ is the usual Lebesgue space. $\|f\|_{p}$ denotes the norm of $f$ in $L^{p}$. $H^{p}$ is the Hardy subspace of $L^{p}$ (see [9]). If $F$ is a measurable subset of $T, L^{p}(F)$ is the space consisting of those $L^{p}$ functions which vanish a.e. off of $F$. (We will think of the elements of $L^{p}$ as functions in the usual incorrect but harmless way.)

Now suppose that $b$ in $H^{\infty}$ is not the zero function and $\|b\|_{\infty} \leqq 1$. Let $\Delta=\left(1-|b|^{2}\right)^{1 / 2}$. Clearly $0 \leqq \Delta \leqq 1$ a.e. . $E$ will denote the measurable set $\left\{e^{i x}: \Delta\left(e^{i x}\right)>0\right\}$. Let $\mathscr{C}$ denote the Hilbert space $H^{2} \oplus L^{2}(E)$ with the obvious norm. Elements of $\mathscr{C}$ will be written $(f, g)$ where $f \in H^{2}$ and $g \in L^{2}(E) . \quad U$ is the isometry on $\mathscr{C}$ given by $U(f, g)=$ $(\chi f, \chi g) . \quad U_{+}$denotes the unilateral shift on $H^{2}: U_{+} f=\chi f$. Let

$$
M=\left\{(b f, \Delta f): f \in H^{2}\right\} .
$$


$M$ is a closed subspace of $\mathscr{H}$ which is invariant for $U$. Suppose that $K=M^{\perp}$ and $P$ is the projection of $\mathscr{C}$ onto $K$. Let $S=P U \mid K$. $S$ is a completely non-unitary contraction; $I-S^{*} S$ and $I-S S^{*}$ are operators of rank 1. This is a special case of a general construction due to Sz.-Nagy and Foias (see [15], [5]). We refer to $S$ as an operator model.

For any $z$ in $D$, let $k_{z}\left(e^{i x}\right)=\left(1-\bar{z} e^{i x}\right)^{-1} \cdot k_{z}$ is the well known Szegö kernel function in $H^{2}$; it has the reproducing property $f(z)=$ $\left\langle f, k_{z}\right\rangle=\int f \bar{k}_{z} d \sigma, z \in D$ and $f \in H^{2}$.

Now $\left(k_{z}, 0\right)$ is in $\mathscr{H}$ and it is easy to see that the element $H_{z}$ of $\mathscr{X}$ defined by

$$
H_{z}=\left([1-\overline{b(z)} b] k_{z},-\overline{b(z)} \Delta k_{z}\right)
$$

(for $z$ in $D$ ) is orthogonal to $M$. Since $\left(k_{z}, 0\right)-H_{z}$ lies in $M$, we see that $H_{z}$ is the projection of $\left(k_{z}, 0\right)$ onto $K=M^{\top}$. Thus, if $(u, v) \in K$,

$$
u(z)=\left\langle(u, v), H_{z}\right\rangle \text {. }
$$

In particular,

$$
\left\langle H_{w}, H_{z}\right\rangle=(1-\overline{b(w)} b(z))(1-\bar{w} z)^{-1}, z, w \in D .
$$

Let $K_{0}$ denote the smallest subspace of $K$ containing $\left\{H_{z}: z \in D\right\}$.

Lemma 2.1. (i) $K \ominus K_{0}=\left\{(0, v): v \in L^{2}(E)\right.$ and $\left.(0, v) \in K\right\}=$ $\left\{x \in K:\left\|S^{* n} x\right\|=\|x\|\right.$ for $\left.n=0,1,2, \cdots.\right\}$

(ii) If $\int \log \Delta d \sigma=-\infty$, then $K_{0}=K$.

Proof. The first equality of sets in (i) follows immediately from (2.2). The second follows from the fact that if $(u, v)$ is in $K$,

$$
\left\|S^{* n}(u, v)\right\|^{2}=\left\|U_{+}^{* n} u\right\|_{2}^{2}+\|v\|_{2}^{2}
$$

which converges to $\|v\|^{2}$ as $n \rightarrow \infty$.

Now suppose that $K_{0} \neq K$. By (i) there is a nonzero $v$ in $L^{2}(E)$ such that $(0, v) \in K$. Since $K=M^{\perp}$, we see that $0=\langle(b p, \Delta p),(0, v)\rangle=$ $\int p \bar{v} \Delta d \sigma$ for all analytic polynomials $p$. Since $v$ is nonzero, it follows that the polynomials are not dense in $L^{2}(\Delta d \sigma)$. Therefore, Szegö's theorem implies that $\int \log \Delta d \sigma>-\infty\left[9, \mathrm{p}\right.$. 58]. Thus if $\int \log \Delta d \sigma=-\infty$, we must have $K_{0}=K$.

Now suppose that $F_{1}$ and $F_{2}$ are Hilbert spaces. A contraction valued analytic function $\left\{F_{1}, F_{2}, \Psi\right\}$ is a function analytic in $D$ taking values in the space of bounded operators from $F_{1}$ to $F_{2}$ and such that $\|\Psi(z)\| \leqq 1$ for all $z$ in $D . \Psi\left(e^{i x}\right)$ is defined to be the limit 
$\lim _{r \rightarrow 1-} \Psi\left(r e^{i x}\right)$ which exists almost everywhere in the strong operator topology [15].

A factorization of $\Psi$ is a representation

$$
\Psi=\Psi_{2} \Psi_{1}
$$

where $\left\{F_{1}, F_{3}, \Psi_{1}\right\}$ and $\left\{F_{3}, F_{2}, \Psi_{2}\right\}$ are contraction valued analytic functions and $F_{3}$ is some Hilbert space. Since the complex numbers can be viewed as the space of bounded operators on the 1-dimensional Hilbert space $C$, we can consider $b$ as a contraction valued analytic function $\{\boldsymbol{C}, \boldsymbol{C}, b\}$. In particular, if $b=\psi_{2} \psi_{1}$ where $\psi_{1}$ and $\psi_{2}$ are in the unit ball of $H^{\infty}$, we have a special case of (2.4).

In [15] the notion of a regular factorization is defined. We specialize this as follows.

Definition 2.2. Let $b$ be an $H^{\infty}$ function whose modulus is bounded by 1 . A scalar regular factorization of $b$ is a representation $b=\psi_{2} \psi_{1}$ where $\psi_{1}, \psi_{2}$ are in $H^{\infty}$ and $\left|\psi_{1}\left(e^{i x}\right)\right| \in\left\{1,\left|b\left(e^{i x}\right)\right|\right\}$ for almost every $x$.

If $b=\psi_{2} \psi_{1}$ is a scalar regular factorization, let $\Delta_{j}=\left(1-\left|\psi_{j}\right|^{2}\right)^{1 / 2}$ and $E_{j}=\left\{e^{i x}: \Delta_{j}\left(e^{i x}\right)>0\right\}, j=1,2$. It is easy to see that $E_{1} \cap E_{2}$ has measure zero and that the sets $E$ and $E_{1} \cup E_{2}$ are the same modulo a Lebesgue null set. It follows that $\Delta_{1} \Delta_{2}=0$ a.e. and $\Delta=\Delta_{1}+\Delta_{2}$ a.e.. Moreover, $L^{2}(E)=L^{2}\left(E_{1}\right) \oplus L^{2}\left(E_{2}\right)$. (We will use $\oplus$ for both internal and external orthogonal direct sum; which is intended should be clear from the context.) We want to characterize a certain type of invariant subspace for $S^{*}$. We will depend heavily on a result of Sz.Nagy and Foias characterizing all of the invariant subspaces of $S^{*}$.

With each scalar regular factorization $b=\psi_{2} \psi_{1}$ we associate a linear manifold $M\left(\psi_{1}, \psi_{2}\right)$ in $\mathscr{C}$ given by $M\left(\psi_{1}, \psi_{2}\right)=\left\{\left(\psi_{2} u, \bar{\psi}_{1} \Delta_{2} u+\right.\right.$ $v): u \in H^{2}$ and $\left.v \in L^{2}\left(E_{1}\right)\right\}$. Since $\left|\psi_{1}\right|=1$ a.e. on $E_{2}$ and $\Delta_{2}=0$ a.e. on $E_{1}$, we have $\left\|\left(\psi_{2} u, \bar{\psi}_{1} \Delta_{2} u+v\right)\right\|^{2}=\left\|\psi_{2} u\right\|_{2}^{2}+\left\|\bar{\psi}_{1} \Delta_{2} u+v\right\|_{2}^{2}=\left\|\psi_{2} u\right\|_{2}^{2}+$ $\left\|\Delta_{2} u\right\|_{2}^{2}+\|v\|_{2}^{2}=\|u\|_{2}^{2}+\|v\|_{2}^{2}$. Hence $M\left(\psi_{1}, \psi_{2}\right)$ is closed. In addition, $M \subset M\left(\psi_{1}, \psi_{2}\right)$ and $M\left(\psi_{1}, \psi_{2}\right)$ is invariant for $U$, so that $\mathscr{H} \ominus M\left(\psi_{1}, \psi_{2}\right)$ is an invariant subspace for $S^{*}$.

The next Lemma is implicitly contained in a proof by de Branges and Rovnyak (see [2], Theorem 6). We include a proof here for completeness. In general (unless otherwise noted), the projection of a Hilbert space onto a subspace $B$ will be denoted by $P_{B} \cdot I_{B}$ is the identity operator on $B$.

LenMa 2.3. Let $H$ be a Hilbert space, $V$ an isometry on $H$ and $A$ and invariant subspace for $V$ such that $A \cap \operatorname{Ker} V^{*}=\{0\}$. Let $B=$ $A^{\perp}$ and $V_{B}$ be the compression $V_{B}=P_{B} V \mid B$. Then $\operatorname{rank}\left(I_{B}-V_{B}^{*} V_{B}\right)=$ 
$\operatorname{dim}(A \ominus V A)$.

Proof. First note that $V_{B}^{*}=V^{*} \mid B$. Let $Q=A \ominus A V$ and $C=$ $\left\{x: V^{*} x \in B\right\}$. Since $(V \mid A)^{*}=P_{A} V^{*} \mid A$, one easily sees that $C=B \oplus Q$. We need two other facts, the first of which is this: $\operatorname{Ker}\left(I_{B}-V_{B}^{*} V_{B}\right)=$ $\{x \in B: V x \in B\}$. To see this, suppose that $x=V_{B}^{*} V_{B} x$ so that $\|x\|^{2}=$ $\left\|V_{B} x\right\|^{2}$. Since $V_{B}=P_{B} V \mid B$, it must be the case that $V x$ is in $B$, which establishes one half of the assertion. If, conversely, $V x$ is in $B$, then $V_{B} x=V x$, so $V_{B}^{*} V_{B} x=V^{*} V x=x$ and $x$ is in $\operatorname{Ker}\left(I_{B}-V_{B}^{*} V_{B}\right)$ as desired.

The second fact is the following: $\{x \in B: V x \in B\}=B \ominus V^{*} Q$. For if $x$ is in $B \ominus V^{*} Q$, then $V x$ is orthogonal to $Q$. However $V x$ is in $C$ (since $V^{*} V=I$ ) and we know that $C=B \oplus Q$, so $V x \in B$ and half of the assertion is proved. The reverse inclusion is clear.

If we put all of this together we have Range $\left(I_{B}-V_{B}^{*} V_{B}\right)=\overline{V^{*} Q}$, so $\operatorname{rank}\left(I_{B}-V_{B}^{*} V_{B}\right)=\operatorname{dim} V^{*} Q$. But $Q \cap \operatorname{Ker} V^{*}=\{0\}$, so $\operatorname{dim} V^{*} Q=$ $\operatorname{dim} Q$ and the proof is complete.

Now suppose that $F$ is a separable Hilbert space. We will denote by $L_{F}^{2}$ the space of (weakly) measurable functions $f$ on $\boldsymbol{T}$ with values in $F$ and such that

$$
\int_{0}^{2 \pi}\left\|f\left(e^{i x}\right)\right\|_{F}^{2} d \sigma(x)<\infty \text {. }
$$

$L_{F}^{2}$ is a Hilbert space with inner product

$$
\langle f, g\rangle=\int_{0}^{2 \pi}\left\langle f\left(e^{i x}\right), g\left(e^{i x}\right)\right\rangle_{F} d \sigma(x) .
$$

$H_{F}^{2}$ is the Hardy subspace of $L_{F}^{2}$ (see [8], [15]). Obviously $L_{C}^{2}=L^{2}$.

If $B$ is a weakly measurable essentially bounded function on $T$ whose values are bounded operators on $F$, then $B f$ will denote the function with values $B\left(e^{i x}\right) f\left(e^{i x}\right)$ whenever $f \in L_{F^{\prime}}^{2}$. We will write $B L_{F^{\prime}}^{2}$ for $\left\{B f: f \in L_{F}^{2}\right\}$ which is contained in $L_{F}^{2}$.

We can now given the main result of this section.

Proposition 2.4. Suppose that $\log \Delta$ is not Lebesgue integrable. Let $N$ be an invariant subspace for $S^{*}$ and let $S_{1}$ be the compression $S_{1}=P_{N} S\left|N=P_{.} U\right| N$. If

$$
\operatorname{rank}\left(I_{N}-S_{i} S_{1}\right)=1 \text {, }
$$

then $N=\mathscr{C} \ominus M\left(\psi_{1}, \psi_{2}\right)$ for some scalar regular factorization $b=$ $\psi_{2} \psi_{1}$ of $b$.

Proof. Suppose that $\left\{C, F, \Psi_{1}\right\}$ and $\left\{F, C, \Psi_{2}\right\}$ are contraction valued analytic functions such that $b=\Psi_{2} \Psi_{1}$. Let $\Delta_{1}\left(e^{i x}\right)=\left(I_{C}-\right.$ 
$\left.\Psi_{1}\left(e^{i x}\right) * \Psi_{1}\left(e^{i x}\right)\right)^{1 / 2}$ and $\Delta_{2}\left(e^{i x}\right)=\left(I_{F}-\Psi_{2}\left(e^{i x}\right)^{*} \Psi_{2}\left(e^{i x}\right)\right)^{1 / 2}$. Now recall that $E=\left\{e^{i x}: \Delta\left(e^{i x}\right)>0\right\}$ so that the closure $\overline{\Delta L^{2}}$ is exactly $L^{2}(E)$. Let

\section{$Z: \mathscr{C} \rightarrow H^{2} \oplus \overline{\Delta_{2} L_{F}^{2}} \oplus \overline{\Delta_{1} L^{2}}$}

denote the mapping defined on the dense subset $H^{2} \oplus \Delta L^{2}$ of $\mathscr{Y}$ by $Z(u, \Delta v)=\left(u, \Delta_{2} \Psi_{1} v, \Delta_{1} v\right) . \quad Z$ is isometric [15, p. 277].

Now since $N$ is invariant for $S^{*}$, a general theorem of Sz.-Nagy and Foias [15, p. 278] says there exists a factorization $b=\Psi_{2} \Psi_{1}$ as above which is regular, i.e., it has the following properties:

(i) The mapping $Z$ is onto.

(ii) $Z N=\left(H^{2} \oplus \overline{\Delta_{2} L_{F}^{2}} \oplus\{0\}\right) \ominus\left\{\left(\Psi_{2} u, \Delta_{2} u, 0\right): u \in H_{F}^{2}\right\}$.

It is also clear that $Z U=V Z$ where $V$ is the isometry on $H^{2} \oplus$ $\overline{\Delta_{2} L_{F}^{2}} \oplus \overline{A_{1} L^{2}}$ given by $V(u, v, w)=(\chi u, \chi v, \chi w)$.

Now suppose that $(u, v) \in(\mathscr{\mathscr { C }} \ominus N) \cap \operatorname{Ker} U^{*}$. Then $0=U^{*}(u$, $v)=\left(U_{+}^{*} u, \bar{\chi} v\right)$, so that $u=c=$ constant and $v=0$. Suppose that $c \neq 0$. Since $\mathscr{H} \ominus N$ is invariant for $U$, it contains the subspace generated by $\left\{U^{n}(c, 0): n=0,1, \cdots\right\}$, namely $H^{2} \oplus\{0\}$. Thus $N \subset\{0\} \oplus L^{2}(E)$ so that $S^{*} \mid N$ is isometric. Since $\int \log \Delta d \sigma=-\infty$, we can conclude from Lemma 2.1 that $N=\{0\}$ which contradicts (2.5). Thus it must be the case that $c=0$ and so $(\mathscr{H} \ominus N) \cap \operatorname{Ker} U^{*}=\{0\}$. We can now invoke (2.5) and Lemma 2.3 to conclude that $\operatorname{dim}[(\mathscr{H} \Theta$ $N) \ominus U(\mathscr{H} \ominus N)]=1$. Equivalently, if $G=Z(\mathscr{H} \ominus N)$, then $\operatorname{dim}(G \ominus V G)=1$. One easily checks that $\left\{\left(\Psi_{2} x, \Delta_{2} x, 0\right): x \in F\right\}$ is contained in $G \ominus V G$. Thus the mapping $x \rightarrow\left(\Psi_{2} x, \Delta_{2} x, 0\right)$ is an isometry of $F$ into $G \ominus V G$. It follows that $\operatorname{dim} F=1$, so we can take $F=C$ and $\Psi_{1}$ and $\Psi_{2}$ to be complex valued (from now on we call them $\psi_{1}$ and $\psi_{2}$, respectively, to emphasize this).

It is shown in [15, p. 290] that under these conditions $b=\psi_{2} \psi_{1}$ is a scalar regular factorization. Thus $M\left(\psi_{1}, \psi_{2}\right)$ makes sense and contains $\left\{\left(\psi_{2} u, \bar{\psi}_{1} \Delta_{2} u+\Delta_{1} v\right): u \in H^{2}\right.$ and $\left.v \in L^{2}\right\}$ as a dense subset. Since $\left|\psi_{1}\right|=1$ a.e. on $E_{2}$ and $\Delta=\Delta_{1}+\Delta_{2}$ a.e., it follows that $Z$ maps this dense subset onto the dense subset $\left\{\left(\psi_{2} u, \Delta_{2} u, \Delta_{1} v\right): u \in H^{2}\right.$ and $\left.v \in L^{2}\right\}$ of $Z(\mathscr{C} \ominus N)$. Hence $M\left(\psi_{1}, \psi_{2}\right)=Z^{-1} Z\left(\mathscr{C}^{\prime} \ominus N\right)=\mathscr{C} \ominus N$. This completes the proof.

Remark 2.5. Suppose that $N=\mathscr{C} \ominus M\left(\psi_{1}, \psi_{2}\right)$ where $b=\psi_{2} \psi_{1}$ is a scalar regular factorization of $b$. Since $N \subset K$, we have $P_{N} P=$ $P_{N}$, so $P_{N} H_{w}=P_{N} P\left(k_{w}, 0\right)=P_{N}\left(k_{w}, 0\right), w \in D$.

We leave it to the reader to verify that for each $w$ in $D$, the projection of $\left(k_{w}, 0\right)$ onto $M\left(\psi_{1}, \psi_{2}\right)$ is exactly $\left(\overline{\psi_{2}(w)} \psi_{2} k_{w}, \overline{\psi_{2}(w)} \bar{\psi}_{1} \Delta_{2} k_{w}\right)$, so that

$$
P_{N} H_{w}=\left(\left[1-\overline{\psi_{2}(w)} \psi_{2}\right] k_{w},-\overline{\psi_{2}(w) \psi_{1}} \Delta_{2} k_{w}\right)
$$




\section{Hence}

$$
\left\langle P_{N} H_{w}, H_{z}\right\rangle=\frac{1-\overline{\psi_{2}(w)} \psi_{2}(z)}{1-\bar{w} z}
$$

for all $z$ and $w$ in $D$.

Now let $\alpha$ and $c$ be as in the introduction and suppose that $\beta$ is the function $\beta(x)=(\alpha(x)-i / 2)(\alpha(x)+i / 2)^{-1}, 0 \leqq x \leqq 1$. Clearly $|\beta|=1$ a.e. For the rest of $\S 2$ and 3 we will assume that $b$ is related to $\alpha$ and $c$ by

$$
b(z)=\exp \left\{(1-z) \int_{0}^{1} \frac{1-\beta(x)}{\beta(x)-z}|c(x)|^{2} d x\right\}, z \in D .
$$

One easily checks that

$$
|b(z)|=\exp \left\{\left(1-|z|^{2}\right) \int_{0}^{1} \frac{\operatorname{Re} \beta(x)-1}{|\beta(x)-z|^{2}}|c(x)|^{2} d x\right\}<1 .
$$

We can thus apply the preceding results in this section to this particular $b$.

Recall the definition of the measure $\nu$ in the Introduction.

LEMMA 2.6. $\int \log \Delta d \sigma=-\infty$ if and only if (1.1) holds.

Proof. The function $\beta$ maps $[0,1]$ into $\boldsymbol{T}-\{1\}$; write $\beta(x)=e^{i \theta(x)}$ where $\theta:[0,1] \rightarrow(0,2 \pi)$. Let $\mu$ be the measure on $(0,2 \pi)$ given by

$$
\mu(F)=\int_{\theta^{-1}(F)}|c|^{2} d m
$$

for every Borel subset of $(0,2 \pi)$. A change of variables [7, p. 163] in (2.8) then gives

$$
|b(z)|=\exp \left\{\int_{0}^{2 \pi} \frac{1-|z|^{2}}{\left|e^{i t}-z\right|^{2}}(\cos t-1) d \mu(t)\right\}, z \in D .
$$

We recognize $\left(1-|z|^{2}\right)\left|e^{i t}-z\right|^{-2}$ as the Poisson kernel; if we set $z=r e^{i x}$ and let $r \rightarrow 1$, Fatou's Theorem implies that $\left|b\left(e^{i x}\right)\right|=$ $\exp [(\cos x-1)(d \mu / d \sigma)(x)]$ a.e. This equation, the fact that $\Delta=$ $\left(1-|b|^{2}\right)^{1 / 2}$, and the elementary inequality $t e^{-t} \leqq\left(1-e^{-t}\right) \leqq t(t \geqq 0)$ together imply that $\log \Delta$ is $\sigma$-integrable if and only if $\log [(1-$ $\cos x)(d \mu / d \sigma)(x)]$ is $\sigma$-integrable.

Now let $\tau:(-\infty, \infty) \rightarrow(0,2 \pi)$ be defined by $e^{i \tau(x)}=(x-i / 2)(x+$ $i / 2)^{-1}$. Thus $\theta=\tau \circ \alpha$, so that $\nu(F)=\mu(\tau(F))$ for any Borel subset of the reals. By the chain rule we have 


$$
2 \pi \tau^{-1^{\prime}}(y) \frac{d \nu}{d n}\left(\tau^{-1}(y)\right)=\frac{d \mu}{d \sigma}(y) \text { a.e. . }
$$

Now $\tau^{-1^{\prime}}(y)=4^{-1}(1-\cos y)^{-1}$ so we find that

$$
\int_{0}^{2 \pi} \log \left[(1-\cos y) \frac{d \mu}{d \sigma}(y)\right] d y=\int_{0}^{2 \pi} \log \left[\frac{\pi}{2} \frac{d \nu}{d n}\left(\tau^{-1}(y)\right)\right] d y .
$$

Making the change of variables $y=\tau(x)$ and using the relation $\tau^{\prime}(x)=$ $\left(x^{2}+1 / 4\right)^{-1}$ yields the equation

$$
\begin{aligned}
& \int_{0}^{2 \pi} \log \left[(1-\cos y) \frac{d \mu}{d \sigma}(y)\right] d y \\
= & 2 \pi \log \frac{\pi}{2}+\int_{-\infty}^{\infty} \log \frac{d \nu}{d n}(x) \frac{d x}{x^{2}+\frac{1}{4}} .
\end{aligned}
$$

The lemma easily follows.

We would like to have a simple way of ensuring that log $\Delta$ is not $\sigma$-integrable. The next proposition gives a useful criterion.

Proposition 2.7. Suppose that $\Phi$ is a positive Baire function on $(-\infty, \infty)$ such that

$$
\int_{0}^{1} \frac{\Phi(\alpha(t))}{1+|\alpha(t)|^{2}}|c(t)|^{2} d t<\infty
$$

and

$$
\int_{-\infty}^{\infty} \frac{\log \Phi(y)}{y^{2}+1} d y=+\infty
$$

Then $\log \Delta$ is not $\sigma$-integrable.

Proof. The composition $\Phi \circ \alpha$ is measurable since $\Phi$ is a Baire function. Assume now that (i) holds. By a change of variables we have

$$
\begin{aligned}
\int_{0}^{1} \frac{\Phi(\alpha(t))}{1+|\alpha(t)|^{2}}|c(t)|^{2} d t & =\int_{-\infty}^{\infty} \frac{\Phi(y)}{1+y^{2}} d \nu(y) \\
& \geqq \int_{-\infty}^{\infty} \Phi(y) \frac{d \nu}{d n}(y) \frac{d y}{1+y^{2}} .
\end{aligned}
$$

It follows from the inequality of the geometric and arithmetic means $[12$, p. 61] that this last integral is not exceeded by

$$
\pi \exp \left\{\frac{1}{\pi} \int_{-\infty}^{\infty} \log \Phi(y) \frac{d y}{1+y^{2}}+\frac{1}{\pi} \int_{-\infty}^{\infty} \log \frac{d \nu}{d n}(y) \frac{d y}{1+y^{2}}\right\} .
$$


If we also assume that (ii) holds, it must be the case that (1.1) holds also. By Lemma 2.6 this is clearly equivalent to the desired conclusion.

Consider, as examples, the functions $\Phi(x)=e^{|x|}$ and $\Phi(x)=\exp \left(|\lambda-x|^{-1}\right)$ where $\lambda$ is a fixed real number. One might choose the first if $|\alpha|$ is not too large to often; the second if the values of $\alpha$ are not heavily concentrated near $\lambda$.

3. When $A$ is completely non-selfadjoint. Assume in this section that $\alpha, c$ and $A$ are as in the introduction and that (1.1) holds. $b$ will be related to $\alpha$ and $c$ by (2.7).

Now suppose that $z$ is not in the essential range of $\alpha$. For each $t$ in $[0,1]$ let

$$
\phi_{t}(z)=\exp \left\{i \int_{0}^{t}(\alpha(x)-z)^{-1}|c(x)|^{2} d x\right\}
$$

REMARK 3.1. If $z$ is not in the essential range of $\alpha$, then $(A-z)^{-1}$ exists and

$$
\begin{array}{r}
{\left[(A-z)^{-1} f\right](x)=\frac{f(x)}{\alpha(x)-z}-i \frac{\phi_{x}(z)^{-1}}{\alpha(x)-z} c(x) \int_{0}^{x} \frac{\phi_{t}(z)}{\alpha(t)-z} \overline{c(t)} f(t) d t,} \\
0 \leqq x \leqq 1 .
\end{array}
$$

The proof is a simple computation using Fubini's Theorem and the fact that $(d / d t) \phi_{t}(z)^{-1}=-i \phi_{t}(z)^{-1}(\alpha(t)-z)^{-1}|c(t)|^{2}$. See also [3].

Recall that $\beta=(\alpha-i / 2)(\alpha+i / 2)^{-1}$, and $|\beta|=1$ a.e. .

Definition 3.2. For each $z$ in $D$ and $t$ in $[0,1]$ let

$$
b_{t}(z)=\exp \left\{(1-z) \int_{0}^{t} \frac{1-\beta(x)}{\beta(x)-z}|c(x)|^{2} d x\right\}
$$

and

$$
Y_{z}(t)=\frac{\beta(t)-1}{1-\beta(t) \bar{z}} \overline{b_{t}(z)} c(t) .
$$

We observe that $b_{1}=b$ and that each $b_{t}$ is in the unit ball of $H^{\infty}$. Moreover, $\left|Y_{z}(t)\right| \leqq K|c(t)|$ where $K$ is a positive constant depending only on $z$. Hence $Y_{z} \in L^{2}(0,1)$ for each $z$ in $D$.

From Remark 2.1 it is clear that $(A+i / 2)^{-1}$ exists and that $(A+i / 2)^{-1} L^{2}(0,1) \subset \mathscr{D}(A)$. It follows that $(A+i / 2) \mathscr{D}(A)=L^{2}(0,1)$. Hence $A$ is a maximal dissipative operator and the discussion in $\S 1$ applies to $A$. In particular, $T=(A-i / 2)(A+i / 2)^{-1}$ is an everywhere defined contraction on $L^{2}(0,1)$. 
REMARK 3.3. For each $t$ in $[0,1]$, let $M_{t}$ be the multiplication operator on $L^{2}(0,1)$ defined by $M_{t}: f \rightarrow \mathfrak{X}_{[0, t]} f$ where $\mathfrak{X}_{[0, t]}$ is the characteristic function of the interval $[0, t] . M_{t}$ is a projection and its range, which we denote by $L^{2}(0, t)$, is the subspace of those functions in $L^{2}(0,1)$ which vanish a.e. on $(t, 1]$.

Let $A_{t}$ and $T_{t}$ be the compressions $A_{t}=M_{t} A \mid L^{2}(0, t)$ and $T_{t}=$ $M_{t} T \mid L^{2}(0, t)$. It is easy to check (using Remark 3.1) that $A_{t}$ is maximal dissipative and $(2 i)^{-1}\left(A_{t}-A_{t}^{*}\right)$ extends to an operator of rank 1. Moreover, $T_{t}=\left(A_{t}-i / 2\right)\left(A_{t}+i / 2\right)^{-1}$. It follows from [15, p. 348] that $I_{t}-T_{t}^{*} T_{t}$ and $I_{t}-T_{t} T_{t}^{*}$ have rank 1 . Here $I_{t}$ is the identity on $L^{2}(0, t)$. This can also be shown from the following proposition.

Proposition 3.4.

$$
(T f)(x)=\beta(x) f(x)+Y_{0}(x) \int_{0}^{x} \overline{c(t)} \overline{b_{t}(0)^{-1}}(\beta(t)-1) f(t) d t
$$

and

$$
\left.\left(T^{*} f\right)(x)=\overline{\beta(x)} f(x)+c(x) b_{x}(0)^{-1} \overline{(\beta(x)}-1\right) \int_{x}^{1} \overline{Y_{0}(t)} f(t) d t
$$

for all $f$ in $L^{2}(0,1)$.

The proof of this is an easy computation using the form of $(A+i / 2)^{-1}$ and the fact that $\phi_{t}(-i / 2)=\overline{b_{t}(0)^{-1}}$.

We will need the following technical lemmas in order to characterize the completely non-selfadjoint subspace of $A$. $m$ will denote Lebesgue measure on $[0,1]$.

Lemma 3.5. If $0 \leqq s<t \leqq 1$ and $z, w \in D$, then

$$
\int_{s}^{t} Y_{w} \bar{Y}_{z} d m=\frac{\overline{b_{s}(w)} b_{s}(z)-\overline{b_{t}(w)} b_{t}(z)}{1-\bar{w} z}
$$

Proof. Using the fact that $|\beta|=1$ a.e. and some computation, it is not hard to show that

$$
\frac{d}{d x}\left[\overline{b_{x}(w)} b_{x}(z)(\bar{w} z-1)^{-1}\right]=Y_{w}(x) \overline{Y_{z}(x)} .
$$

The Lemma follows upon integrating this equation from $s$ to $t$.

Lемma 3.6. If $0<|z|<1$, then

$$
\int_{0}^{1} \overline{Y_{z}(t)}(\overline{\beta(t)}-1) b(0) b_{t}(0)^{-1} c(t) d t=z^{-1}(b(z)-b(0)) .
$$

Proof. One verifies that 


$$
b_{t}(z) b_{t}(0)^{-1}=\exp \left\{z \int_{0}^{t} \frac{(\overline{\beta(x)}-1)^{2}}{1-\overline{\beta(x)} z}|c(x)|^{2} d x\right\}
$$

Differentiating (with $z \neq 0$ ) gives

$$
\frac{d}{d t}\left(z^{-1} b_{t}(z) b_{t}(0)^{-1}=\overline{Y_{z}(t)}(\overline{\beta(t)}-1) b_{t}(0)^{-1} c(t),\right.
$$

$0 \leqq t \leqq 1$. If we multiply this equation by $b(0)$, integrate from 0 to 1 and recall that $b_{1}=b$, we find that the equation in the statement of the Lemma is true.

LEMMA 3.7.

$$
\int_{0}^{x}\left|(\beta(t)-1) b_{t}(0)^{-1} c(t)\right|^{2} d t=\left|b_{x}(0)\right|^{-2}-1,0 \leqq x \leqq 1 .
$$

Proof. We easily check that

$$
\left|b_{t}(0)\right|^{-2}=\exp \left\{-2 \int_{0}^{t}(\operatorname{Re} \beta(x)-1)|c(x)|^{2} d x\right\},
$$

so that

$$
\frac{d}{d t}\left|b_{t}(0)\right|^{-2}=2(1-\operatorname{Re} \beta(t))\left|b_{t}(0)\right|^{-2}|c(t)|^{2} .
$$

Now $|\beta-1|^{2}=2(1-\operatorname{Re} \beta)$ a.e. (since $|\beta|=1$ a.e.); substituting this in the previous equation and integrating from 0 to $x$ gives the desired conclusion.

Now let $K$ and $S$ be the Hilbert space and operator, respectively, associated with $b$ as in $\S 2$. We define a linear mapping $W_{0}$ from finite linear combinations of $\left\{H_{z}: z \in D\right\}$ into $L^{2}(0,1)$ by $W_{0}\left(\sum c_{j} H_{z_{j}}\right)=$ $\sum c_{j} Y_{z_{j}}, z_{j} \in D$ and $c_{j}$ complex.

Lemma 3.8. (i) $W_{0}$ extends in a unique way to an isometry $W$ from $K$ into $L^{2}(0,1)$.

(ii) $\left\langle W^{*} g, H_{z}\right\rangle=\int_{0}^{1} g \bar{Y}_{z} d m, g \in L^{2}(0,1)$ and $z \in D$.

Proof. If $z, w \in D$, we see from (2.3) and Lemma 3.5 with $s=0, t=1$, that

$$
\begin{aligned}
\left\langle W_{0} H_{w}, W_{0} H_{z}\right\rangle & =\int_{0}^{1} Y_{w} \bar{Y}_{z} d m \\
& =\left\langle H_{w}, H_{z}\right\rangle .
\end{aligned}
$$

Thus $W_{0}$ preserves inner products and hence norms. Since we are assuming that (1.1) holds, Lemma 2.1 (ii) and Lemma 2.6 imply that 
$\left\{H_{z}: z \in D\right\}$ spans $K$. Thus $W_{0}$ has a unique isometric extension $W$ to all of $K$, so that (i) follows. (ii) is clear from the definition of $W_{0}$ and the proof is complete.

Note that the vector $(b, \Delta)$ in $\mathscr{H}$ spans $M \ominus U M$. It follows that $U^{*}(b, \Delta)$ lies in $M^{\perp}=K$.

Lemma 3.9. Let $f \in K$. Then $\|S f\|=\|f\|$ if and only if $f$ is orthogonal to $U^{*}(b, \Delta)$.

Proof. $S$ is the compression of the isometry $U$ the subspace $K=$ $M^{\perp}$. It follows from the proof of Lemma 2.3 that $\{f \in K:\|S f\|=$ $\|f\|\}=K \ominus U^{*}(M \ominus U M)$. One easily checks that the vector $(b, \Delta)$ spans $M \ominus U M$, which completes the proof.

The following theorem identifies the completely non-unitary subspace of T. Assertions (i), (iii) and (iv) were known (up to Cayley transforms) to Brodskii and Livsic, although they did not identify the subspace $W K$ as the range of an isometry. Their proof used an argument about the resolvent of $A$ which does not seem to work when $A$ is unbounded. The following proof relates $W, S$ and $T$ in a natural way and has the advantage of working when the spectrum of $T$ is the entire unit circle.

THEOREM 1. (i) WK is a reducing subspace for $T$.

(ii) $W S=T W$.

(iii) $T \mid W K$ is completely non-unitary.

(iv) $T \mid(W K)^{\perp}$ is unitary.

Proof. First we show that $S^{*}=W^{*} T^{*} W$. For this it will suffice to show that $S^{*}$ and $W^{*} T^{*} W$ agree on the total subset $\left\{H_{z}: z \in D\right\}$ of $K$. Recall that the isometry $U$ acting on $\mathscr{C}$ is exactly $U^{+} \oplus M_{\chi}$ where $M_{\chi}: f \rightarrow \chi f$ acts on $L^{2}(E)$ and $U_{+}$is the unilateral shift on $H^{2}$. Now $\left(U_{+}^{*} f\right)(z)=z^{-1}\left(f(z)-(f(0))\right.$ if $f \in H^{2}$, and $S^{*}=U^{*} \mid K$. It follows from an easy computation that

$$
S^{*} H_{z}=\bar{z} H_{z}-\overline{b(z)} U^{*}(b, \Delta), z \in D .
$$

Now in the expression for $T^{*}$ given in Proposition 3.4, replace $f$ by $Y_{z}$ and use Lemma 3.5 to get

$$
\left(T^{*} Y_{z}\right)(x)=\overline{\beta(x)} Y_{z}(x)+c(x) b_{x}(0)^{-1}(\overline{\beta(x)}-1)\left(\overline{b_{x}(z)} b_{x}(0)-\overline{b(z)} b(0)\right) .
$$

Using this, the definition of $Y_{z}$, and the fact that $|\beta|=1$ a.e., we easily compute that

$$
\left(T^{*} Y_{z}\right)(x)=\bar{z} Y_{z}(x)-\overline{b(z)}\left[c(x)(\overline{\beta(x)}-1) b(0) b_{x}(0)^{-1}\right] .
$$


For convenience, let $h(x)=b(0) c(x)(\overline{\beta(x})-1) b_{x}(0)^{-1}$ We have just shown that

$$
T^{*} Y_{z}=\bar{z} Y_{z}-\overline{b(z)} h, z \in D .
$$

Applying $W^{*}$ to this equation and recalling that $W H_{z}=Y_{z}$, we have

$$
W^{*} T^{*} W H_{z}=\bar{z} H_{z}-\overline{b(z)} W^{*} h, z \in D \text {. }
$$

A comparison on this with (3.1) shows that we must prove that $W^{*} h=U^{*}(b, \Delta)$. By Lemma 3.8 (ii), the definition of $h$ and Lemma 3.6 ,

$$
\begin{aligned}
\left\langle W^{*} h, H_{z}\right\rangle & =z^{-1}(b(z)-b(0)) \\
& =\left(U_{+}^{*} b\right)(z) \\
& =\left\langle U^{*}(b, \Delta), H_{z}\right\rangle,
\end{aligned}
$$

$z \neq 0$. Since the functions $\left\{H_{z}: z \in D\right.$ and $\left.z \neq 0\right\}$ span $K$, we have $W^{*} h=U^{*}(b, \Delta)$ as desired. Hence

$$
S^{*}=W^{*} T^{*} W \text {. }
$$

Now we shall show that $W K$ is invariant for $T^{*}$. Since $\left\{Y_{z}: z \in\right.$ $D$ s spans $W K$, it is enough to show that $T^{*} Y_{z}$ is in $W K$ for each $z$ in $D$. The action of $T^{*}$ on $Y_{z}$ is given by (3.2); from this it is clear that we need only argue that $h \in W K . W$ is an isometry, so $h$ will lie in $W K$ if and only if $\left\|W^{*} h\right\|=\|h\|$. We know that $W^{*} h=$ $U^{*}(b, \Delta)$; an easy computation shows that $\left\|W^{*} h\right\|^{2}=\left\|U^{*}(b, \Delta)\right\|^{2}=$ $1-|b(0)|^{2}$. On the other hand, it follows from Lemma 3.7 and the definition of $h$ that $\|h\|^{2}=\int|h|^{2} d m=1-|b(0)|^{2}=\left\|W^{*} h\right\|^{2}$. Thus $W K$ is invariant for $T^{*}$.

Now $W W^{*}$ is the projection of $L^{2}(0,1)$ onto $W K$. Denote this projection by $E$. Since $W K$ is invariant for $T^{*}$, we can let $W$ act on equation (3.4) from the left to get $W S^{*}=E T^{*} W=T^{*} W$. Therefore $W$ provides a unitary equivalence between $S^{*}$ and $T^{*} \mid W K$.

Let $B=E T \mid W K$, so that $B^{*}=T^{*} \mid W K$. Clearly $B$ and $S$ are unitarily equivalent by way of $W$ :

$$
W S=B W .
$$

We have shown that $W U^{*}(b, \Delta)=h$. It follows from Lemma 3.9 that $g$ in $W K$ is orthogonal to $h$ if and only if $\|B g\|=\|g\|$. For such a $g$ we have $\|g\|=\|B g\|=\|E T g\| \leqq\|T g\| \leqq\|g\|$. Hence $\|E T g\|=\|T g\|$ so that $T g \in W K$. Thus $T(W K \ominus\{h\}) \subset W K$. In order to conclude that $W K$ is invariant for $T$, we need only show that $T h \in W K$.

From the definition of $h$, Proposition 3.4, Lemma 3.7 and some 
computation we have

$$
\begin{aligned}
(T h)(x) & =b(0) \beta(x)(\overline{\beta(x)}-1) b_{x}(0)^{-1} c(x) \\
& +b(0)(\beta(x)-1) \overline{b_{x}(0)} c(x)\left(\left|b_{x}(0)\right|^{-2}-1\right) \\
& =-b(0) Y_{0}(x)
\end{aligned}
$$

i.e.,

$$
T h=-b(0) Y_{0} .
$$

Since $Y_{0} \in W K$ we have shown that $T W K \subset W K$. Thus $W K$ reduces $T$.

It follows that $B=T \mid W K$ which implies that (3.5) can be improved to $W S=T W . T \mid W K$ is therefore unitarily equivalent to $S$ and so is completely non-unitary.

Finally, we know from Remark 3.3 that $I-T^{*} T$ and $I-T T^{*}$ have 1-dimensional range. Setting $z=0$ in (3.2) yields $T^{*} Y_{0}=$ $-\overline{b(0) h}$. Combining this with (3.6) shows that $\left(I-T^{*} T\right) h=\left(1-|b(0)|^{2}\right) h$ and $\left(I-T T^{*}\right) Y_{0}=\left(1-|b(0)|^{2}\right) Y_{0}$. The ranges of the operators $I-$ $T^{*} T$ and $I-T T^{*}$ are therefore contained in $W K$ so their kernels contain $(W K)^{\perp}$. It follows that $T \mid(W K)^{\perp}$ is unitary. This completes the proof.

We are now in a position to decide when the subspace $W K$ is all of $L^{2}(0,1)$. We will need a simple lemma (see [11, Lemma 3.3] for the proof) and a definition.

Lemma 3.10. Let $H_{1}$ and $H_{2}$ be Hilbert spaces and let $V: H_{1} \rightarrow H_{2}$ be an isometry. Suppose that $E$ is a projection in $H_{2}$ and $V^{*} E V$ is a projection in $H_{1}$. Then $V H_{1}$ is invariant for $E$.

DeFinition 3.11. Let $b_{t}$ be as in Definition 3.2 and define $q_{t}$ by $b=b_{t} q_{t}, 0 \leqq t \leqq 1$. $\left\{b_{t}\right\}$ will be called a regular family if $b=b_{t} q_{t}$ is a scalar regular factorization for each $t$ in $[0,1]$.

Theorem 2. $W K=L^{2}(0,1)$ if and only if $|c|>0$ a.e. and $\left\{b_{t}\right\}$ is a regular family.

Proof. Suppose first that $W K=L^{2}(0,1)$ and $M_{t}$ is as in Remark 3.3. Then $P_{t}=W^{*} M_{t} W$ is a projection in $K$ since $M_{t}$ is a projection in $L^{2}(0,1)$. Let $K_{t}=P_{t} K$; clearly $K_{t}=W^{*} M_{t} L^{2}(0,1)=W^{*} L^{2}(0, t)$, $0 \leqq t \leqq 1$. Now $L^{2}(0, t)$ is easily seen to be invariant for $T^{*}$, so, by Theorem 1 (ii), $K_{t}$ is invariant for $S^{*}$.

Let $S_{t}$ be the compression $S_{t}=P_{t} S \mid K_{t}$ and $T_{t}$ be as in Remark 3.3. It follows from Theorem 1 (ii) that $W$ provides a unitary equivalence between $S^{*} \mid K_{t}$ and $T^{*} \mid L^{2}(0, t)$, or, equivalently, that $S_{t}$ and $T_{t}$ are unitarily equivalent. Thus, by Remark 3.3, we have rank $\left(I_{K_{t}}-S_{t}^{*} S_{t}\right)=1$. We can now invoke Proposition 2.4 to conclude that 
$K_{t}=\mathscr{H} \ominus M\left(\psi_{1}, \psi_{2}\right)$ for some scalar regular factorization $b=\psi_{2} \psi_{1}$. Now, by Lemma 3.5 we have

$$
\begin{aligned}
\left\langle P_{t} H_{w}, H_{z}\right\rangle & =\left\langle M_{t} W H_{w}, W H_{z}\right\rangle \\
& =\int_{0}^{t} Y_{w} \bar{Y}_{z} d m \\
& =\left(1-\overline{b_{t}(w)} b_{t}(z)\right)(1-\bar{w} z)^{-1}, z, w \in D .
\end{aligned}
$$

On the other hand, since $K_{t}=\mathscr{H} \ominus M\left(\psi_{1}, \psi_{2}\right)$, equation (2.6) implies that

$$
\left\langle P_{t} H_{w}, H_{z}\right\rangle=\left(1-\overline{\psi_{2}(w)} \psi_{2}(z)\right)(1-\bar{w} z)^{-1}, z, w \in D .
$$

Comparing (3.7) and (3.8) shows that $b_{t}=a \psi_{2}$ for some constant $a$ of modulus 1. This clearly implies that $b=b_{t} q_{t}$ is a scalar regular factorization. Since $t$ is arbitrary in $[0,1]$, we have shown that $b_{t}$ is a regular family.

Now let $F=\{x: c(x)=0\}$. It is clear from Definition 3.2 that each $Y_{z}$ vanishes a.e. on $F$. Since the functions $Y_{z}$ span $W K=$ $L^{2}(0,1)$, it must be the case that $F$ has Lebesgue measure zero. This completes the proof one way.

Conversely, suppose that $\left\{b_{t}\right\}$ is a regular family and $|c|>0$ a.e. Let $b=b_{t} q_{t}$ define $q_{t}$ and set $K_{t}=\mathscr{H} \ominus M\left(b_{t}, q_{t}\right), 0 \leqq t \leqq 1 . \quad P_{t}$ will denote the projection of $K$ onto $K_{t}$. Again by (2.6) we have

$$
\left\langle P_{t} H_{w}, H_{z}\right\rangle=\left(1-\overline{b_{t}(w)} b_{t}(z)\right)(1-\bar{w} z)^{-1}, z, w \in D .
$$

On the other hand, we can use Lemma 3.5 as in equation (3.7) to conclude that

$$
\left\langle W^{*} M_{t} W H_{w}, H_{z}\right\rangle=\left(1-\overline{b_{t}(w)} b_{t}(z)\right)(1-\bar{w} z)^{-1}, z, w \in D .
$$

Comparing this with (3.9) and recalling that $\left\{H_{z}: z \in D\right\}$ spans $K$ shows that $P_{t}=W^{*} M_{t} W$. Therefore, by Lemma 3.10, $W K$ is invariant for $M_{t}, 0 \leqq t \leqq 1$. Moreover, $Y_{0}$ is in $W K$, so if $0 \leqq s<t \leqq 1$ and $\mathfrak{X}_{(s t]}$ is the characteristic function of the interval $(s, t], \mathfrak{X}_{(s, t]} Y_{0}$ is exactly $M_{t} Y_{0}-M_{s} Y_{0}$ which must lie in $W K$. It follows that $p Y_{0}$ is in $W K$ for any step function $p$. If $g$ is orthogonal to $W K$, then $\int p Y_{0} \bar{g} d m=0$ for all step functions $p$. Consequently $Y_{0} \bar{g}=0$ a.e. Since $\beta$ never takes the value 1 and $|c|>0$ a.e., it follows from Definition 3.2 that $\left|Y_{0}\right|>0$ a.e. Thus $g=0$ a.e. and $W K=L^{2}(0,1)$. This completes the proof.

We would like to have a condition on the pair $(\alpha, c)$ that is equivalent to the hypothesis of Theorem 2. To this end suppose that $|c|>0$ a.e. and let $\rho$ be the measure on $[0,1]$ given by $\rho(F)=$ $\int_{F}|c|^{2} d m$. It is clear that $\rho$ is mutually absolutely continuous with 
respect to Lebesgue measure $m$. Thus for any $y$ in the essential range of $\alpha$ (which we denote by $R(\alpha)$ ) and any real $t$, define $\eta(y, t)$ by

$$
\eta(y, t)=\lim _{\delta \rightarrow 0} \frac{\rho\left(\alpha^{-1}(y-\delta, y+\delta) \cap[0, t]\right)}{\rho\left(\alpha^{-1}(y-\delta, y+\delta)\right)}
$$

It will follow from the proof of Lemma 3.14 that for each $t$, this limit exists for almost all $y$ in the set $\sigma_{a c}(\alpha)$ defined below.

Definition 3.12. Suppose that $F$ is a measurable subset of $R(\alpha)$. $\alpha$ will be called essentially invertible on $F$ (with respect to the measure $\rho)$ if for each $t$ in $[0,1], \eta(y, t) \in\{0,1\}$ for almost every $y$ in $F$.

Essential invertibility is a kind of measure-theoretic one-to-oneness condition. To see this assume that $\alpha$ is essentially invertible on $F$. For each rational $r$ in $[0,1]$ there exists a set $N_{r}$ of measure zero contained in $R(\alpha)$ such that $\eta(y, r)$ exists and lies in $\{0,1\}$ for all $y$ in $F-N_{r}$. Let $N$ denote the union of all of these sets $N_{r} . \quad N$ has measure zero and $\eta(y, r)$ exists and lies in $\{0,1\}$ for each $y$ in $F-N$ and rational $r$.

For a fixed $y$ in $F-N, \eta(y, r)$ is a nondecreasing function of $r$ ( $r$ rational). Let $x=\sup \{r: r$ is rational and $\eta(y, r)=0\}$. Clearly $\eta(y, r)=0$ if $r<x$ and $\eta(y, r)=1$ if $r>x$. From the definition of $\eta(y, t)$ it is clear that the sets $\alpha^{-1}(y-\delta, y+\delta), \delta>0$, are concentrated around $x$ as $\delta \rightarrow 0$. Accordingly, $x$ is called the essential pre-image of $y$.

DEFINITION 3.13. The absolutely continuous spectrum of $\alpha$ is the set

$$
\sigma_{a c}(\alpha)=\left\{y: \lim _{\tilde{c} \rightarrow 0}(2 \delta)^{-1} m\left(\alpha^{-1}(y-\delta, y+\delta)\right) \text { exists and is positive. }\right\}
$$

Note that $\sigma_{a c}(\alpha) \subset R(\alpha)$ and that the limit in the definition agrees almost everywhere with the Radon-Nikodym derivative $d\left(m \alpha^{-1}\right) / d n$; here $m \alpha^{-1}$ is the measure given by $\left(m \alpha^{-1}\right)(F)=m\left(\alpha^{-1}(F)\right)$.

Lemma 3.14. Suppose that $|c|>0$ a.e.. Then $\left\{b_{t}\right\}$ is a regular family if and only if $\alpha$ is essentially invertible on $\sigma_{a c}(\alpha)$.

Proof. The function $\beta$ maps $[0,1]$ into $\boldsymbol{T}-\{1\}$. Write $\beta(x)=$ $e^{i \theta(x)}$ where $\theta:[0,1] \rightarrow(0,2 \pi)$. For $0 \leqq t \leqq 1$ let $\nu_{t}$ and $\mu_{t}$ be the measures on $(-\infty, \infty)$ and $(0,2 \pi)$, respectively, given by $\nu_{t}(F)=\rho([0$, t] $\left.\cap \alpha^{-1}(F)\right)$ and $\mu_{t}(G)=\rho\left([0, t] \cap \theta^{-1}(G)\right)$. An argument analogous to that in Lemma 2.6 implies that

$$
\left|b_{t}\left(e^{i x}\right)\right|=\exp \left[(\cos x-1) \frac{d \mu_{t}}{d \sigma}(x)\right] \text { a.e. . }
$$


Thus the condition that $\left\{b_{t}\right\}$ be a regular family is exactly the condition that for any $t, 0 \leqq t \leqq 1$,

$$
\frac{d \mu_{t}}{d \sigma}(x) \in\left\{0, \frac{d \mu_{1}}{d \sigma}(x)\right\} \text { a.e. . }
$$

As in Lemma 2.6 we compute

$$
\frac{d \nu_{t}}{d n}(x)=\frac{1}{2 \pi} \frac{d \mu_{t}}{d \sigma}(\tau(x)) \cdot \tau^{\prime}(x)
$$

$x$ real. Since $\tau^{\prime}(x)$ never vanishes and $\nu_{1}=\nu,(3.10)$ is equivalent to

$$
\frac{d \nu_{t}}{d n}(x) \in\left\{0, \frac{d \nu}{d n}(x)\right\} \text { a.e. . }
$$

Since $\rho$ and $m$ are mutually absolutely continuous, it follows that $\{x:(d \nu / d n)(x)>0\}$ and $\sigma_{a c}(\alpha)$ differ only by a Lebesgue null set. Moreover, $0 \leqq d \nu_{t} / d n \leqq d \nu / d n$, so (3.11) holds automatically for almost all $x$ outside of $\sigma_{a c}(\alpha)$. Hence for $\left\{b_{t}\right\}$ to be a regular family it is necessary and sufficient that for each $t$,

$$
\frac{d \nu_{t}}{d n}(x) \frac{d \nu}{d n}(x)^{-1} \in\{0,1\}
$$

for almost all $x$ in $\sigma_{a c}(\alpha)$. Since, for each $t$ in $[0,1],\left(d \nu_{t} / d n\right)(x)=$ $\lim _{\delta \rightarrow 0}(2 \delta)^{-1} \rho\left(\alpha^{-1}(x-\delta, x+\delta) \cap[0, t]\right)$ for almost all $x$, we see that this is equivalent to the condition that $\alpha$ be essentially invertible on $\sigma_{a c}(\alpha)$. This completes the proof.

Since $A$ is maximal dissipative, we know from Theorem 1 and the discussion in $\S 1$ that $W K$ reduces $A, A \mid W K$ is completely nonselfadjoint and $A \mid(W K)^{\perp}$ is selfadjoint. Putting this together with Theorem 2 and Lemma 3.14 yields our main theorem.

THEOREM 3. A is completely non-selfadjoint if and only if $|c|>$ 0 a.e. and $\alpha$ is essentially invertible (with respect to $\rho$ ) on $\sigma_{\alpha c}(\alpha)$.

Corollary 3. Suppose that $|c|>0$ a.e. and $\alpha$ is monotone. Then $A$ is completely non-selfadjoint.

Proof. Let $t \in[0,1]$ and assume that $\alpha$ is nondecreasing. If $y<$ $\alpha(t)$, then $\alpha^{-1}(y-\delta, y+\delta)$ is contained in $[0, t]$ if $\delta$ is small enough. Hence $\eta(y, t)=1$. Similarly $\eta(y, t)=0$ if $y>\alpha(t)$. Thus $\alpha$ is essentially invertible on $R(\alpha)$ which contains $\sigma_{a c}(\alpha)$. The same conclusion holds if $\alpha$ is nonincreasing. Therefore, if $|c|>0$ a.e., Theorem 3 implies that $A$ is completely non-selfadjoint. 
The next corollary follows immediately from Theorem 3 .

COROLlaRY 3.16. If $|c|>0$ a.e. and $\sigma_{a c}(\alpha)$ has measure zero, then $A$ is completely non-selfadjoint.

The reader can check $\sigma_{a c}(\alpha)$ has measure zero if and only if $b$ is an inner function. This will certainly happen if, e.g., $\alpha$ has countable range.

CoRollary 3.17. Suppose that $c$ and 1/c are essentially bounded and that $\alpha$ is continuously differentiable. Then $A$ is completely nonselfadjoint if and only if $\alpha$ is monotone.

This is an easy consequence of Theorem 3 and the definition of essential invertibility. The hypothesis can be weakened in several obvious ways. We leave the proof for the reader.

We conclude this section with a rather curious result on the perturbation of singular spectral multiplicity.

Corollary 3.18. Let $B_{1}=\int \lambda d E_{1}(\lambda)$ and $B_{2}=\int \lambda d E_{2}(\lambda)$ be bounded selfadjoint operators on a separable Hilbert space. Suppose that $B_{1}$ and $B_{2}$ have no point spectra and no absolutely continuous spectra. Suppose further that the spectral measures $E_{1}$ and $E_{2}$ are mutually absolutely continuous, that is, $E_{1}(G)=0$ if and only if $E_{2}(G)=$ 0 for $G$ a Borel subset of the line. Then, given $\varepsilon>0$, there exists a compact operator $K$ with $\|K\|<\varepsilon$ such that $B_{1}+K$ and $B_{2}$ are unitarily equivalent. Moreover, $K$ is contained in each Schatten p-class $C_{p}$ for $p>1$.

Proof. We will need the fact, which is probably part of the folklore, that any selfadjoint operator $B$ with no point spectrum can be represented as a multiplication operator $M_{\phi}: f \rightarrow \phi f$ acting on $L^{2}(\alpha$, $b$ ), where $[a, b]$ is a given interval and $\phi$ is in $L^{\infty}(a, b)$. One way to see this is to decompose $B$ as direct sum of at most countably many selfadjoint operatorators $\left\{B_{k}\right\}$, each of which has a cyclic vector. $B_{k}$ can be represented as a multiplication $f(\lambda) \rightarrow \lambda f(\lambda)$ on $L^{2}\left(\mu_{k}\right)$ for some finite positive measure $\mu_{k}$ with compact support on the line. Now for each $B_{k}$, select a non-degenerate subinterval $I_{k}$ of $[a, b]$ in such a way that the $I_{k}$ 's are disjoint and their union is $[a, b]$. We may assume that the total mass of $\mu_{k}$ equals the length of $I_{k}$. $\mu_{k}$ has no atoms, so we can choose a strictly increasing function (as in the proof of Theorem 5) $\dot{\phi}_{k}: I_{k} \rightarrow(-\infty, \infty)$ such that $m \phi_{k}^{-1}=\mu_{k}$, where $m$ is Lebesgue measure. The map $f \rightarrow f \circ \phi_{k}$ from $L^{2}\left(\mu_{k}\right)$ to $L^{2}\left(I_{k}\right)$ is clearly an isometry. It is onto since $\dot{\phi}_{k}$ is strictly increasing, and so 
induces a unitary equivalence between $M_{\phi_{k}}$ and $B_{k}$. Define $\phi$ on [ $\alpha$, $b]$ so that its restriction to $I_{k}$ is $\phi_{k}$. $M_{\phi}$ is the desired operator. If $E(G)$ is the spectral projection for $B$ corresponding to a Borel set $G$, then $E(G)$ corresponds to the map $f \rightarrow \chi_{\phi^{-1}(G)} f$ on $L^{2}(a, b)$.

We apply this as follows. Represent $B_{1}$ and $B_{2}$ as $M_{\alpha_{1}}$ and $M_{\alpha_{2}}$, respectively, acting on $L^{2}(0,1)$. By our assumption about $E_{1}$ and $E_{2}$, the measures $m \alpha_{k}^{-1}$ are mutually absolutely continuous. Let $g$ be the Radon-Nikodym derivative of $m \alpha_{1}^{-1}$ with respect to $m \alpha_{2}^{-1}$, so that $m \alpha_{1}^{-1}(G)=\int_{G} g d\left(m \alpha_{2}^{-1}\right)=\int_{\alpha_{2}^{-1}(G)} g\left(\alpha_{2}(x)\right) d x$. Now $g \geqq 0$ and $g \circ \alpha_{2}$ vanishes only on a set of Lebesgue measure zero. Let $c_{1} \equiv 1$ and $c_{2}=\left(g \circ \alpha_{2}\right)^{1 / 2}$ and $\nu_{k}(G)=\int_{\alpha_{\bar{k}}^{1}(G)}\left|c_{k}\right|^{2} d m$. In the theory developed above $\nu_{k}$ corresponds to the operator $A_{k}=M_{\alpha_{k}}+i V_{k}$, where $\left(V_{k} f\right)(x)=c_{k}(x) \int_{0}^{x} \overline{c_{k}(t)} f(t) d t$. We have just shown that $\nu_{1}=\nu_{2}$. It follows that for $k=1,2$, the functions $b_{k}$ associated with $\alpha_{k}$ and $c_{k}$ as in (2.7) are identical. Since $B_{1}$ and $B_{2}$ have purely singular spectra, $\nu_{1}=\nu_{2}$ is a singular measure. It follows that $\sigma_{a c}\left(\alpha_{k}\right)$ has measure zero, so that the operator $W_{k}$ : $K_{k} \rightarrow L^{2}(0,1)$ is onto for $k=1,2$ by Corollary 3.16. Therefore $\left(A_{k}\right.$ $i / 2)\left(A_{k}+i / 2\right)^{-1}$ is unitarily equivalent to $S_{k}$ for $k=1,2$. Now $b_{1}=$ $b_{2}$, so that $S_{1}=S_{2}$; hence $A_{1}$ and $A_{2}$ are unitarily equivalent, say $A_{1}=$ $U A_{2} U^{-1}$ for $U$ unitary. Therefore $M_{\alpha_{1}}+D=U M_{\alpha_{2}} U^{-1}$ where $D=i\left(V_{1}-\right.$ $\left.U V_{2} U^{-1}\right)$. It is easy to see that $V_{2}$ is unitarily equivalent to the Volterra operator $V_{1}$ which is well known to be in the Schatten $p$ class $C_{p}$ for $p>1$. Therefore $D$ is in $C_{p}$ and $\|D\| \leqq 2\left\|V_{1}\right\|$.

Now, choose $a>2\left(\left\|V_{1}\right\| / \varepsilon\right)$ and apply the above discussion to $a B_{1}$ and $a B_{2}$ rather than $B_{1}$ and $B_{2}$, and then divide by $a$. Since $\left\|a^{-1} D\right\|<\varepsilon$, we are done if we set $K=a^{-1} D$.

4. Related results for almost unitary contractions. The techniques in the preceding sections can be used to study other integral operators. Suppose, for example, that $A>0$ and $a:[0, A] \rightarrow[0,2 \pi)$ is measurable. Let $X$ be the operator on $L^{2}(0, A)$ given by

$$
(X f)(x)=\xi(x) f(x)-\int_{0}^{x} e^{(t-x) / 2} \xi(t) f(t) d t
$$

where $\xi(x)=e^{i \alpha(x)}$. Let $X_{0}$ denote this operator when $a(x) \equiv 0$ and let $M_{\xi}$ be the multiplication $M_{\xi}: f \rightarrow \xi f$. Clearly $X=X_{0} M_{\xi}$.

It is easy to compute that $X$ is a contraction and, in fact, that $I-X^{*} X$ and $I-X X^{*}$ are positive rank-one operators. For $0 \leqq t \leqq$ $A$, we define $X_{t}$ (analogous to $T_{t}$ in Remark 3.3) to be the compression of $X$ to $L^{2}(0, t)$. It is easy to compute that $I_{t}-X_{t}^{*} X_{t}=\left\langle\cdot, u_{t}\right\rangle u_{t}$ and $I_{t}-X_{t} X_{t}^{*}=\left\langle\cdot, v_{t}\right\rangle v_{t}$, where $I_{t}$ is the identity on $L^{2}(0, t), u_{t}(x)=$ $\xi(x) \exp ((x-t) / 2)$ and $v_{t}(x)=\exp (-x / 2), 0 \leqq x \leqq t$. Another compu- 
tation shows that $X^{*} v_{A}=e^{-A / 2} u_{A}$ and $X u_{A}=e^{-A / 2} v_{A}$ so that $u_{A}$ and $v_{A}$ play the roles of $h$ and $Y_{0}$, respectively, in Theorem 1 .

We associate with $X$ the functions $\left\{b_{t}\right\}$ in the unit ball of $H^{\infty}$ given by

$$
b_{t}(z)=\exp \left\{-\frac{1}{2} \int_{0}^{t} \frac{\xi(x)+z}{\xi(x)-z} d x\right\}, 0 \leqq t \leqq A, z \in D .
$$

Set $b=b_{A}$ and associate $S$ and $K$ with $b$ as in $\S 2$.

For each $z$ in $D$ let

$$
h_{z}(t)=\overline{b_{t}(z)}(1-\xi(t) \bar{z})^{-1}, 0 \leqq t \leqq 1, z \in D .
$$

Define $V_{0}$ from finite linear combinations of $\left\{H_{z}: z \in D\right\}$ (in $K$ ) into $L^{2}(0, A)$ by

$$
V_{0}\left(\sum c_{j} H_{z_{j}}\right)=\sum c_{j} h_{z_{j}}, z_{j} \in D
$$

We define essential invertility for the function $a$ as in Definition 3.12 but with $\rho$ replacted by Lebesgue measure $m$. Let $\mu$ be the measure on $[0,2 \pi)$ given by $\mu(F)=m\left(a^{-1}(F)\right)$. The arguments of the previous sections, altered only in computational details, yield the following theorem.

Theorem 4. Suppose that

$$
\int\left(\log \frac{d \mu}{d \sigma}\right) d \sigma=-\infty
$$

Then the mapping $V_{0}$ has a unique isometric extension $V$ from $K$ into $L^{2}(0, A) . \quad V K$ reduces $X, X \mid(V K)^{\perp}$ is unitary and $X \mid V K$ is completely non-unitary. $V S=X V$, so that $X \mid V K$ is unitarily equivalent to $S$. $V K=L^{2}(0, A)$ if and only if $\left\{b_{t}\right\}$ is a regular family, which is the case if and only if $a$ is essentially invertible on $\sigma_{a c}(a)$.

In the case $a \equiv 0$, the mapping $V$ is equivalent to one used by Sarason to study the Volterra integration operator [12]. Note that in this case $b(z)$ reduces to inner function

$$
\exp \left(-\frac{A}{2} \frac{1+z}{1-z}\right)
$$

and Theorem 4 implies that $V K=L^{2}(0, A)$.

The operators $S$ of $\S 2$ are known to represent a certain abstract class of contractions. Using this fact and Theorem 4 we can prove the following representation theorem. This may be considered as an analog, for contractions, of the triangular model of Brodskii and Livsic [3]. $K_{0}$ will denote the compact operator 


$$
K_{0}: f(x) \longrightarrow \int_{0}^{x} \exp \left(\frac{t-x}{2}\right) f(t) d t
$$

so that $X_{0}=I-K_{0}$.

THEOREM 5. Let $T$ be a contraction operator on a Hilbert space $H$ such that $I-T^{*} T$ and $I-T T^{*}$ are rank one operators. Suppose that $T^{*}$ has no isometric restriction and that the spectrum of $T$ is contained in the unit circle. Let $A=-\log \left(1-\left\|I-T^{*} T\right\|\right)$. Then there exists a non-decreasing function $a:[0, A] \rightarrow[0,2 \pi)$ with this property: if $\xi(x)=e^{i a(x)}$, then $T$ is unitarily equivalent to $\left(I-K_{0}\right) M_{\hat{\xi}}$ acting on $L^{2}(0, A)$.

Proof. Let $T$ be as in the hypotheses of the Theorem. $T$ is completely non-unitary (otherwise $T^{*}$ would have an isometric part) so by results of Sz.-Nagy and Foias [15], $T$ is unitarily equivalent to an operator $S$ acting on $K$ as in $\S 2$. Let $b$ be the associated $H^{\infty}$ function. Since $\boldsymbol{T}$ contains the spectrum of $S$ (by hypothesis), $b$ has no zeros in $D$ (see [15, p. 247]). Since $|b|$ is bounded by $1, b$ has a representation of the form

$$
b(z)=\exp \left\{-\frac{1}{2} \int_{0}^{2 \pi} \frac{e^{i x}+z}{e^{i x}-z} d \mu(x)\right\}, z \in D,
$$

where $\mu$ is a finite positive measure on $[0,2 \pi)$. (see [9, p. 63]).

Set $A=\mu([0,2 \pi))$ and let $\alpha:[0, A] \rightarrow[0,2 \pi)$ be a nondecreasing function such that $\mu(F)=m\left(a^{-1}(F)\right)$ for every Borel subset of $[0,2 \pi)$. Here $m$ is Lebesgue measure on $[0, A]$. (It will suffice to take $a(t)=$ $\inf \{x: \mu([0, x]) \geqq t\}$.) By a change of variable in (4.3) we have

$$
b(z)=\exp \left\{-\frac{1}{2} \int_{0}^{A} \frac{\xi(x)+z}{\xi(x)-z} d x\right\}, z \in D,
$$

where $\xi(x)=e^{i a(x)}$. Let $b_{t}$ be defined as in (4.2) and suppose that $V$ is associated with $\left\{b_{t}\right\}$ as in Theorem 4 . We want to conclude that $S$ is unitarily equivalent to $X=\left(I-K_{0}\right) M_{\hat{s}}$ acting on $L^{2}(0, A)$.

Since $a$ is monotone we can invoke the argument in Corollary 3.15 to establish the essential invertibility of $a$ on $\sigma_{a c}(a)$. Furthermore, the condition in Theorem 4 that

$$
\int\left(\log \frac{d \mu}{d \sigma}\right) d \sigma=-\infty
$$

is used only to show that the span $K_{0}$ of $\left\{H_{z}: z \in D\right\}$ is all of $K$. Since $S^{*} \mid K \ominus K_{0}$ is the maximal isometric part of $S^{*}$ (see Lemma 2.1), we see from our hypothesis on $T^{*}$ that $K=K_{0}$ automatically. Hence 
Theorem 4 is applicable and the operators $T, S$ and $X=\left(I-K_{0}\right) M_{\xi}$ are all unitarily equivalent.

Finally, from our previous discussion $I-X^{*} X=\left\langle\cdot, u_{A}\right\rangle u_{A}$, so $\left\|I-T^{*} T\right\|=\left\|I-X^{*} X\right\|=\left\|u_{A}\right\|^{2}=1-e^{-A}$. Hence $A=-\log (1-$ $\left.\left\|I-T^{*} T\right\|\right)$. This completes the proof.

We can use Theorem 5 to extend some results of Ahern and Clark [1]. For the rest of this section $T$ will be a contraction satisfying the hypothesis of Theorem 5 .

Let $W$ acting on $N \supset H$ be the minimal strong unitary dilation of $T$ ([6], [15]), i.e. $W$ is unitary, $T^{n}=P_{H} W^{n} \mid H$, and $T^{* n}=P_{I I} W^{-n} \mid H$, $n \geqq 0$. For any continuous function $u$ on the unit circle, $u(W)$ makes sense as a normal operator on $N . T_{u}$ will be the operator on $H$ defined by $T_{u}=P_{H} u(W) \mid H$. If $u$ is in $H^{\infty}$, then $T_{u}=u(T)$ where the last operator is taken in the sense of the Sz.-Nagy and Foias operational calculus [15].

The corollaries that follow were proved by Ahern and Clark [1] under the additional hypothesis that $T^{* n} \rightarrow 0$ strongly (this happens if and only if $b$ is an inner function). [1] also contains an analogue of Theorem 5 for this case.

CoRollary 4.1. Suppose that $Z$ is a unitary operator such that

$$
\left(I-K_{0}\right) M_{\xi}=Z T Z^{*} \text {. }
$$

where $M_{\bar{s}}$ is as in Theorem 5. Then

$$
u\left(M_{\xi}\right)+K=Z T_{u} Z^{*}
$$

for some compact $K$.

Proof. The important part of Theorem 5 (for the purposes of this proof) is that $T$ is unitarily equivalent to $Y+K_{1}$ where $Y$ is unitary and $K_{1}$ is compact. An argument in [1] then shows that the same unitary equivalence takes $T_{u}$ onto $u(Y)+K$ for some compact $K$. This completes the proof.

Recall that the Fredholm spectrum of an operator $B$ is the set $s p_{F}(B)=\{\lambda: B-\lambda$ is not Fredholm $\}$. The Weyl spectrum $w(B)$ is the intersection $w(B)=\cap\{s p(B+K): K$ is compact $\}$. The index of Fredholm operator $B$ is the integer $i(B)=\operatorname{dim}(\operatorname{Ker} B)-\operatorname{dim}\left(\operatorname{Ker} B^{*}\right)$. It is known that

$$
w(B)=\operatorname{sp}_{F}(B) \cup\{\lambda: B-\lambda \text { is Fredholm and } i(B-\lambda) \neq 0\} .
$$

The reader can find these definitions and facts in [13] and [14]. 
Now suppose that $b$ is as in Theorem 5 , so $b$ has the the representation (4.3). It follows from [15, p. 247] that $s p(T)=s p(S)$ is exactly the closed support of $\mu$, which is equal to the essential range of $\xi$ (where $\mu$ is considered as a measure on $T$ ).

CoRollaRy 4.2. $\quad w\left(T_{u}\right)=s p_{F}\left(T_{u}\right)=u(s p(T))$

Proof. Let $\xi$ be as in Theorem 5 and recall that the property of being Fredholm is invariant under compact perturbations. From Theorem 5 and Corollary 5.1 we have $s p_{F}\left(T_{u}\right)=s p_{F}\left(u\left(M_{\xi}\right)+K\right)=$ $s p_{F}\left(u\left(M_{\xi}\right)\right)$.

Now $u\left(M_{\xi}\right)=M_{u \circ \xi}$ is a multiplication operator on a non-atomic measure space and hence $s p_{F}\left(u\left(M_{\xi}\right)\right)=s p\left(u\left(M_{\xi}\right)\right)$. It follows that $s p_{F}\left(u\left(M_{\xi}\right)\right)=u($ essential range $\xi)=u(s p(T))$. Finally, if $T_{u}-\lambda$ is Fredholm, then $i\left(T_{u}-\lambda\right)=i\left(u\left(M_{\xi}\right)+K-\lambda\right)=i\left(u\left(M_{\xi}\right)-\lambda\right)=0$; this follows from the fact that the index does not change under compact perturbation and $u\left(M_{\xi}\right)-\lambda$ is normal. Thus $w\left(T_{u}\right)=s p_{F}\left(T_{u}\right)$. This completes the proof.

CoRollary 5.3. $T_{u}$ is compact if and only if $u$ vanishes on $\operatorname{sp}(T)$.

Proof. Let $K$ be compact. $u\left(M_{\xi}\right)+K$ is compact if and only if $u\left(M_{\xi}\right)=M_{u \circ \xi}$ is compact, which can happen only when $M_{u \circ \xi}=0$, i.e. $u(\xi(x))=0$ a.e. . This is the case if and only if $u$ vanishes on the essential range of $\xi$ which coincides with $s p(T)$. The proof is complete.

Added in proof. (1) Douglas N. Clark has informed me that the converse to Lemma 2.1 (ii) is true. Here is his proof. Define $U: L^{2}\left(\Delta^{2} d \sigma\right) \rightarrow L^{2}(E)$ by $U f=\Delta f . \quad U$ is clearly a unitary operator; hence $\{U p: p$ is a polynomial $\}$ spans $L^{2}(E)$ if and only if the polynomials span $L^{2}\left(\Delta^{2} d \sigma\right)$. The former is true precisely when $K_{0}=K$ (see the proof of Lemma 2.1) whereas the latter is true if and only if $\log \Delta^{2}=2 \log \Delta$ is not integrable, by Szegö's theorem.

(2) In Corollary 3.18, suppose that the spectral measures $E_{1}$ and $E_{2}$ of $B_{1}$ and $B_{2}$, respectively, are assumed only to have the same closed support, rather than to be mutually absolutely continuous. Then $B_{1}$ and $B_{2}$ have the same (essential) spectra and it follows from two famous theorems of von Neumann that $B_{1}+K$ and $B_{2}$ are unitarily equivalent for some compact operator $K$ (see Charakterisierung des Spektrums eines Integraloperators, Actualités Sci. Ind., 229, Paris (1935), p. 11). An improvement of one of von Neumann's theorems (S. Kuroda, On a theorem of Weyl-von Neumann, Proc. Japan Acad. 34 (1958), 11-15) together with a recent refinement of the other 
(P. R. Halmos, Limits of shifts, to appear) shows that the full conclusion of Corollary 3.18 is true with the weaker hypotheses. In fact, $B_{1}$ and $B_{2}$ need not be singular, but only "essential" selfadjoint operators.

\section{REFERENCES}

1. P. R. Ahern and D. N. Clark, On functions orthogonal to invariant subspaces, Acta Math., 124 (1970), 191-204.

2. L. de Branges and J. Rovnyak, Appendix on Square Summable Power Series, Canonical Models in Quantum Scattering Theory, Perturbation theory and its application in quantum mechanics, Wiley, New York, 1966, pp. 295-391.

3. M. S. Brodskii and M. S. Livsic, Spectral analysis of nonselfadjoint operators and intermediate systems, Amer. Math. Soc. Translations, Series 2, Vol. 13, Amer. Math. Soc. Providence, R. I., (1960), 265-346.

4. D. N. Clark, One dimensional perturbations of restricted shifts, J. Analyse Math., 25 (1972), 169-191.

5. R. G. Douglas, Structure theory for operators I, J. Reine Angew. Math., 232 (1968), 180-193.

6. P. R. Halmos, A Hilbert Space Problem Book, D. Van Nostrand, Princeton, N. J., 1967.

7. Measure Theory, D. Van Nostrand, Princeton, N. J., 1950.

8. H. Helson, Lectures on Invariant Subspaces, Academic Press, Princeton, N. J., 1964.

9. K. Hoffman, Banach Spaces of Analytic Funtions, Prentice-Hall, Englewood Cliffs, N. J., 1962.

10. T. L. Kriete, On when an almost selfadjoint operator has no selfadjoint part, Abstract 663-518, Notices Amer. Math. Soc., Vol. 16, No. 1, Jan. (1969), 237.

11. T. L. Kriete, A generalized Paley-Wiener theorem, J. Math. Anal. and Appl., 36 (1971), 529-555.

12. D. Sarason, A remark on the Volterra operator, J. Math. Anal. and Appl., 12 (1965), 244-246.

13. M. Schechter, Invariance of the essential spectrum, Bull. Amer. Math. Soc., 71 (1965), 365-367.

14. R. T. Seeley, The index of elliptic systems of singular integral operators, J. Math. Anal. and Appl., 7 (1963), 289-309.

15. B. Sz.-Nagy and C. Foias, Analyse harmonique des opérateurs de l'espace de Hilbert, Akademiai Kiado, Budapest, 1967.

Received November 12, 1970, and in revised form December 19, 1971. The main ideas of this paper (in the form of Theorem 4 and Theorem 5) are part of the author's 1968 doctoral dissertation done under the direction of Professor Marvin Rosenblum at the University of Virginia. This research supported in part by ONR Contract Nonr 474 (14), and an NSF Institutional Grant at the University of Virginia.

UNIVERSITY OF VIRGINIA 



\section{PACIFIC JOURNAL OF MATHEMATICS}

\section{EDITORS}

\author{
H. SAMELSON \\ Stanford University \\ Stanford, California 94305 \\ C. R. HOBBY \\ University of Washington \\ Seattle, Washington 98105
}

\section{J. DuGundJI}

Department of Mathematics University of Southern California Los Angeles, California 90007

RICHARD ARENS

University of California

Los Angeles, California 90024

\section{ASSOCIATE EDITORS}
E. F. BECKENBACH
B. H. NeumanN
F. WOLF
K. YOSHIDA

\section{SUPPORTING INSTITUTIONS}

\author{
UNIVERSITY OF BRITISH COLUMBIA \\ CALIFORNIA INSTITUTE OF TECHNOLOGY \\ UNIVERSITY OF CALIFORNIA \\ MONTANA STATE UNIVERSITY \\ UNIVERSITY OF NEVADA \\ NEW MEXICO STATE UNIVERSITY \\ OREGON STATE UNIVERSITY \\ UNIVERSITY OF OREGON \\ OSAKA UNIVERSITY
}

\author{
UNIVERSITY OF SOUTHERN CALIFORNIA \\ STANFORD UNIVERSITY \\ UNIVERSITY OF TOKYO \\ UNIVERSITY OF UTAH \\ WASHINGTON STATE UNIVERSITY \\ UNIVERSITY OF WASHINGTON \\ AMERICAN MATHEMATICAL SOCIETY \\ NAVAL WEAPONS CENTER
}

The Supporting Institutions listed above contribute to the cost of publication of this Journal, but they are not owners or publishers and have no responsibility for its content or policies.

Mathematical papers intended for publication in the Pacific Journal of Mathematics should be in typed form or offset-reproduced, (not dittoed), double spaced with large margins. Underline Greek letters in red, German in green, and script in blue. The first paragraph or two must be capable of being used separately as a synopsis of the entire paper. The editorial "we" must not be used in the synopsis, and items of the bibliography should not be cited there unless absolutely necessary, in which case they must be identified by author and Journal, rather than by item number. Manuscripts, in duplicate if possible, may be sent to any one of the four editors. Please classify according to the scheme of Math. Rev. Index to Vol. 39. All other communications to the editors should be addressed to the managing editor, Richard Arens, University of California, Los Angeles, California, 90024.

50 reprints are provided free for each article; additional copies may be obtained at cost in multiples of 50 .

The Pacific Journal of Mathematics is published monthly. Effective with Volume 16 the price per volume (3 numbers) is $\$ 8.00$; single issues, $\$ 3.00$. Special price for current issues to individual faculty members of supporting institutions and to individual members of the American Mathematical Society: $\$ 4.00$ per volume; single issues $\$ 1.50$. Back numbers are available.

Subscriptions, orders for back numbers, and changes of address should be sent to Pacific Journal of Mathematics, 103 Highland Boulevard, Berkeley, California, 94708.

PUBLISHED BY PACIFIC JOURNAL OF MATHEMATICS, A NON-PROFIT CORPORATION

Printed at Kokusai Bunken Insatsusha (International Academic Printing Co., Ltd.), 270, 3-chome Totsuka-cho, Shinjuku-ku, Tokyo 160, Japan. 


\section{Pacific Journal of Mathematics}

\section{Vol. 42, No. $2 \quad$ February, 1972}

Stephen Richard Bernfeld, The extendability of solutions of perturbed scalar differential equations ................................. 277

James Edwin Brink, Inequalities involving $f_{-} p$ and $f^{(n)}{ }_{q}$ for $f$ with $n$

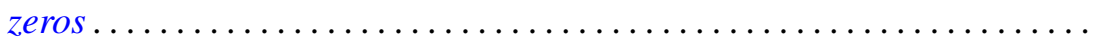

Orrin Frink and Robert S. Smith, On the distributivity of the lattice of filters of a groupoid

Donald Goldsmith, On the density of certain cohesive basic sequences .... 323

Charles Lemuel Hagopian, Planar images of decomposable continua . . . . . 329

W. N. Hudson, A decomposition theorem for biadditive processes ........ 333

W. N. Hudson, Continuity of sample functions of biadditive processes......

Masako Izumi and Shin-ichi Izumi, Integrability of trigonometric series.

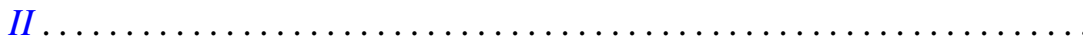

H. M. Ko, Fixed point theorems for point-to-set mappings and the set of

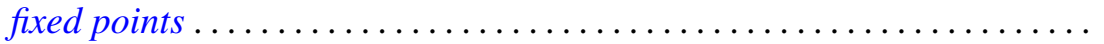

Gregers Louis Krabbe, An algebra of generalized functions on an open interval: two-sided operational calculus ...

Thomas Latimer Kriete, III, Complete non-selfadjointness of almost selfadjoint operators.................................

Shiva Narain Lal and Siya Ram, On the absolute Hausdorff summability of a Fourier series .

Ronald Leslie Lipsman, Representation theory of almost connected groups...

James R. McLaughlin, Integrated orthonormal series.... . .

H. Minc, On permanents of circulants.

Akihiro Okuyama, On a generalization of $\Sigma$-spaces.....

Norberto Salinas, Invariant subspaces and operators of class $(S)$

James D. Stafney, The spectrum of certain lower triangular matrices as operators on the $l_{p}$ spaces .......................

Arne Stray, Interpolation by analytic functions

$\mathrm{Li} \mathrm{Pi} \mathrm{Su}$, Rings of analytic functions on any subset of the complex plane.

R. J. Tondra, A property of manifolds compactly equivalent to compact manifolds.... 